\title{
Defect of SIRT1-FoxO3a axis is associated with the production of reactive oxygen species during protein kinase CK2 downregulation-mediated cellular senescence and nematode aging
}

\author{
Hye-Jun Ham, Jeong-Woo Park \& Young-Seuk Bae* \\ School of Life Sciences, BK21 Plus KNU Creative BioResearch Group, Kyungpook National University, Daegu 41566, Korea
}

\begin{abstract}
We investigated whether SIRT1 is associated with reactive oxygen species (ROS) accumulation during CK2 downregulationmediated senescence. SIRT1 overexpression suppressed ROS accumulation, reduced transcription of FoxO3a target genes, and nuclear export and acetylation of FoxO3a, which were induced by CK2 downregulation in HCT116 and MCF-7 cells. Conversely, overexpression of a dominant-negative mutant SIRT1 (H363Y) counteracted decreased ROS levels, increased transcriptional activity of FoxO3a, and increased nuclear import and decreased acetylation of FoxO3a, which were induced by CK2 upregulation. CK2 downregulation destabilized SIRT1 protein via an ubiquitin-proteasome pathway in human cells, whereas CK2 overexpression reduced ubiquitination of SIRT1. Finally, the SIRT1 activator resveratrol attenuated the accumulation of ROS and lipofuscin as well as lifespan shortening, and reduced expression of the DAF-16 target gene sod-3, which were induced by CK2 downregulation in nematodes. Altogether, this study demonstrates that inactivation of the SIRT1-FoxO3a axis, at least in part, is involved in ROS generation during CK2 downregulationmediated cellular senescence and nematode aging. [BMB Reports 2019; 52(4): 265-270]
\end{abstract}

\section{INTRODUCTION}

Reactive oxygen species (ROS) induce DNA damage, which results in the stabilization of p53 and subsequent overexpression of $\mathrm{p} 21^{\mathrm{Cip} 1 / \mathrm{NAF} 1}$, which then proceeds to inhibit cell cycle

${ }^{*}$ Corresponding author. Tel: +82-53-950-6355; Fax: +82-53-9432762; E-mail: ysbae@knu.ac.kr

https://doi.org/10.5483/BMBRep.2019.52.4.156

Received 12 July 2018, Revised 30 July 2018, Accepted 10 August 2018

Keywords: FoxO3a, Protein kinase CK2, Reactive oxygen species, Senescence, SIRT1 progression. Cellular senescence is the state of irreversible cell cycle arrest at the G1 phase (1-4). We previously reported that downregulation of protein kinase CK2 (CK2) activity induces premature senescence of various human cells through the

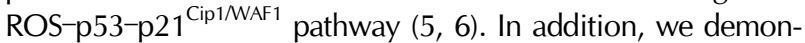
strated that the PI3K-AKT-mTOR pathway is involved in CK2 inhibition-mediated ROS generation (7) and that CK2 downregulation stimulated ROS accumulation via AKTmediated phosphorylation of FoxO3a, which resulted in reduced transcriptional activity of FoxO3a (8). CK2 downregulation resulted in reduced longevity and onset of age-related biomarkers associated with both ROS generation and the AGE-1/PI3K-AKT-1/AKT-DAF-16/FoxO pathway in nematodes (9).

Forkhead box $\mathrm{O}$ (FoxO) class proteins are transcription factors, which modulate expression of various antioxidant genes. Humans express four FoxO class members (FoxO1a, FoxO3a, FoxO4, and FoxO6); of these, FoxO3a is primarily involved in resistance to oxidative stress (10). The transcriptional activity of FoxO3a can be regulated by post-translational modifications such as phosphorylation and acetylation. For example, AKT phosphorylates Ser 253 on FoxO3a, causing nuclear export of FoxO3a (11). The complex of cyclic AMP response element binding protein (CBP) and its associated protein p300 (CBP/p300) acetylates FOXO3a, resulting in the exclusion of FOXO3a from the nucleus (12). It has been demonstrated that the deacetylation of FOXO3a by SIRT1, a paralog of silent information regulator 2 (Sir2), represses the activity of FOXO3a (13). However, SIRT1 has additionally been reported to stimulate the transcription of FoxO3a target genes via deacetylation of FOXO3a (14). Therefore, the detailed mechanism for SIRT1-mediated FoxO3a regulation remains unclear. We investigated the physiological significance of a SIRT1-FoxO3a axis in ROS accumulation during cellular senescence and nematode aging induced by CK2 downregulation. CK2 downregulation mediated inhibition of the SIRT1-FoxO3a axis, which resulted in ROS accumulation. 


\section{RESULTS}

\section{SIRT1 overexpression attenuated ROS production induced by CK2 $\alpha$ knockdown in cells}

To analyze whether SIRT1 is involved in CK2 inhibitionmediated ROS production, HCT116 and MCF-7 cells were transfected with SIRT1 CDNA in the presence of CK2 $\alpha$ siRNA (Supplementary Fig. 1A) and were stained with $\mathrm{CM}-\mathrm{H}_{2}$ DCFDA and dihydroethidium. Consistent with previous studies, CK2 knockdown increased ROS production, as indicated by the right-shift in fluorescence on flow cytometry. Ectopic expression of SIRT1, however, abrogated the induction of ROS levels mediated by CK2 downregulation (Fig. 1A). In contrast, ectopic expression of CK2 $\alpha$ (Supplementary Fig. 1B) reduced ROS generation, but overexpression of a dominant-negative mutant SIRT1 $(\mathrm{H} 363 \mathrm{Y})(15,16)$ or treatment with the SIRT1 inhibitor nicotinamide (15 mM) (Fig. 1B, Supplementary Fig. 1C) suppressed this outcome. These results suggest that decreased SIRT1 activity is involved in the stimulation of ROS production during CK2 downregulation-mediated senescence.

\section{SIRT1 overexpression attenuated CK2 $\alpha$ knockdown-mediated reduction of the FoxO3a transcriptional activity}

To analyze the impact of SIRT1 on the transcription of FoxO3a target genes during CK2 inhibition-mediated senescence, we introduced CK2 $\alpha$ siRNA and/or pECE-Flag-SIRT1 into HCT116 and MCF-7 cells. CK2 $\alpha$ knockdown decreased mRNA levels of major antioxidant genes, including

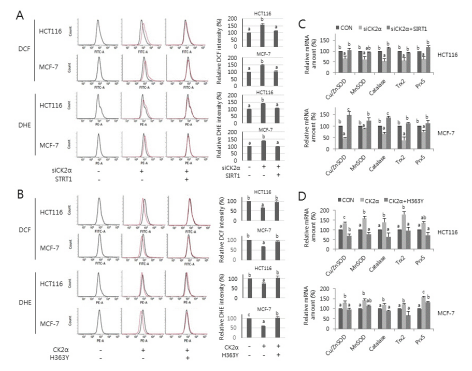

Fig. 1. Effect of SIRT1 activity on ROS production and FoxO3a transcription activity during CK2 downregulation-mediated senescence. HCT116 and MCF-7 cells were treated with CK2 $\alpha$ siRNA (A, C) or pcDNA3.1-HA-CK2 $\alpha$ (B, D) in the absence or presence of pECE-flag-SIRT1 (A, C) or pECE-flag-SIRT1 mutant (H363Y) (B, D) for $48 \mathrm{~h}$. (A, B) The cells were incubated with $5 \mu \mathrm{M}$ CM-H $\mathrm{H}_{2}$ DCFDA (DCF) or $1 \mu \mathrm{M}$ dihydroethidium (DHE), as previously described (6). Fluorescence intensity was determined by flow cytometry analysis. Representative data from three independent experiments are shown (left panels). The graphs show the relative amount of DCF or DHE fluorescence (right panels). (C, D) Total RNA was extracted from the cells and then RT-PCR was performed using specific primers for CK2 $\alpha$, $\mathrm{Cu} / \mathrm{ZnSOD}, \mathrm{MnSOD}$, catalase, thioredoxin-2 (Trx2), and peroxiredoxin-5 (Prx5). $\beta$-actin was used as a loading control. The graphs show the relative mRNA levels of these antioxidant genes.
Cu/ZnSOD, MnSOD, catalase, thioredoxin-2, and peroxiredoxin-5, but ectopic expression of SIRT1 attenuated this result (Fig. 1C, Supplementary Fig. 2A). In contrast, the mRNA levels of these antioxidant genes increased in ectopic CK2 $\alpha$ expressing cells, but concomitant overexpression of SIRT1 mutant (H363Y) (Fig. 1D, Supplementary Fig. 2B) or treatment with $15 \mathrm{mM}$ nicotinamide (Supplementary Fig. 2B) suppressed this event. These results suggest that reduced transcriptional activity of FoxO3a mediated by CK2 downregulation is, at least in part, a result of inhibition of SIRT1 activity.

\section{SIRT1 overexpression attenuated the nuclear export of} FoxO3a induced by CK2 $\alpha$ downregulation in cells We examined whether SIRT1 regulates nuclear localization of FoxO3a during CK2 downregulation-mediated senescence. Immunocytochemical analysis clearly showed that, consistent with a previous study (8), CK2 $\alpha$ knockdown stimulated cytoplasmic sequestration of FoxO3a in cells. Ectopic expression of SIRT1 abolished the CK2 downregulation-mediated nuclear export of FoxO3a (Fig. 2A). Further densitometric analysis of immunofluorescence images confirmed this result. In contrast, the nuclear import of FoxO3a was stimulated in ectopic CK2 $\alpha$ expressing cells, but concomitant overexpression of SIRT1 mutant $(\mathrm{H} 363 \mathrm{Y})$ abrogated FoxO3a nuclear localization in ectopic CK2 $\alpha$ expressing cells (Fig. 2B). Collectively, these data demonstrate that CK2 downregulation results in cytoplasmic retention of FoxO3a by inhibiting SIRT1 in cells.

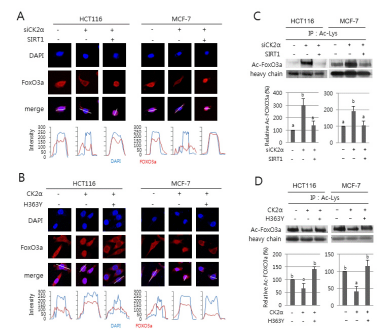

Fig. 2. Effect of SIRT1 activity on the nuclear export and acetylation of FoxO3a during CK2 downregulation-mediated senescence. HCT116 and MCF-7 cells were treated with CK2 $\alpha$ siRNA $(\mathrm{A}, \mathrm{C})$ or pcDNA3.1-HA-CK2 $\alpha$ (B, D) in the absence or presence of pECE-flag-SIRT1 (A, C) or pECE-flag-SIRT1 mutant (H363Y) (B, D) for 48 h. (A, B) The subcellular distribution of FOXO3a (red) was determined by immunofluorescence staining (upper panels). Cells were counterstained with DAPI (blue) to visualize the nuclei. Fluorescence intensity was quantified using ImageJ software (bottom panels). Arbitrary intensity values for FoxO3a (red) or DAPI (blue) are shown relative to the reference line (white) used for analysis. (C, D) Cell lysate was immunoprecipitated (IP) with anti-acetyl Lys antibody, followed by western blotting with anti-FoxO3a antibodies. Cell lysates IP with IgG heavy chain served as a loading control. Representative data from three independent experiments are shown (upper panels). The graphs show the relative amount of acetylated FoxO3a (bottom panels). 


\section{SIRT1 overexpression attenuated FoxO3a acetylation induced by CK2 $\alpha$ knockdown in cells}

It has been reported that the acetylation status of Lys residues on FoxO3a can be modulated by the histone acetyl transferase $\mathrm{CBP} / \mathrm{p} 300$ and the deacetylase SIRT1 (12-14). To determine the effect of SIRT1 on FoxO3a acetylation during CK2 downregulation-mediated senescence, we immunoprecipitated total proteins containing acetylated Lys from cell extracts using anti-acetylated Lys antibodies and then probed the immunoprecipitates with anti-FoxO3a antibodies. Cell extracts immunoprecipitated with IgG were used as a control. Higher levels of acetylated FoxO3a protein were observed in cells downregulating CK2 $\alpha$ compared with control cells. Ectopic expression of SIRT1, however, abrogated the induction in acetylated FoxO3a levels caused by CK2 downregulation (Fig. 2C). In contrast, CK2 $\alpha$ overexpression (Fig. 2D) or treatment with $15 \mathrm{mM}$ nicotinamide (Supplementary Fig. 3) decreased the levels of acetylated FoxO3a proteins, but ectopic expression of SIRT1 mutant (H363Y) suppressed this inhibition. Taken together, these data suggest that CK2 downregulation-mediated reduction in the transcriptional activity of FoxO3a is, at least in part, caused by increased acetylation of FoxO3a via SIRT1 inhibition.

\section{CK2 downregulation induced destabilization of SIRT1 via ubiquitin-proteasome pathway}

It has been previously reported that SIRT1 deacetylase activity can be stimulated by CK2 phosphorylation $(17,18)$. In this study, however, we observed that CK2 regulates the protein levels of SIRT1 (Fig. 3A). To analyze whether CK2 regulates expression of

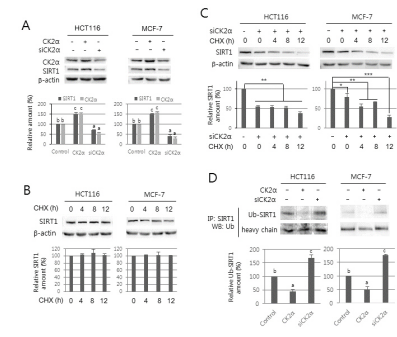

Fig. 3. Effect of CK2 activity on the stability of SIRT1 protein. (A) HCT116 and MCF-7 cells were treated with CK2 $\alpha$ siRNA or pcDNA3.1-HA-CK2 $\alpha$ for $48 \mathrm{~h}$. Cell extracts were electrophoresed on an $8 \%(\mathrm{w} / \mathrm{v})$ SDS-polyacrylamide gel and visualized by western blotting with anti-CK $2 \alpha$ and SIRT1 antibodies. $\beta$-actin was used as a loading control. (B, C) Cells were treated with $50 \mu \mathrm{g} / \mathrm{ml}$ of cycloheximide $(\mathrm{CHX})$ for the indicated times in the absence (B) or presence (C) of CK2 $\alpha$ siRNA. Protein extracts were visualized by western blotting with anti-CK2 $\alpha$ and SIRT1 antibodies. $\beta$-actin was used as a loading control. (D) Cells were treated with CK2 $\alpha$ siRNA or pcDNA3.1-HA-CK2 $\alpha$ for $48 \mathrm{~h}$. Cell lysates were immunoprecipitated (IP) with anti-SIRT1 antibodies followed by western blotting with anti-ubiquitin antibodies. Representative data from three independent experiments are shown (upper panels). The graphs show the relative amount of the indicated proteins (bottom panels). Values indicate mean \pm S.E. ${ }^{*} \mathrm{P}<0.05$; $* * P<0.01 ; * * * P<0.001$.
SIRT1 at the transcription level, we extracted total RNA from cells transfected with CK2 $\alpha$ siRNA or pcDNA3.1-HA-CK2 $\alpha$ and then performed RT-PCR using specific primers for SIRT1. Knockdown or overexpression of CK2 $\alpha$ did not change the mRNA levels of SIRT1 compared with control cells, suggesting that CK2 regulates SIRT1 at a post-transcription level (Supplementary Fig. 4). To investigate whether the CK2 regulates SIRT1 expression at translation or post-translation levels, cells treated with the protein synthesis inhibitor cycloheximide $(50 \mu \mathrm{g} / \mathrm{ml})$ were grown in the absence (Fig. 3B) or presence (Fig. 3C) of CK2 $\alpha$ siRNA. The level of SIRT1 protein steadily decreased after the addition of cycloheximide in the cells downregulating $\mathrm{CK} 2 \alpha$, suggesting that CK2 downregulation destabilizes the SIRT1 protein (Fig. 3B and C). To examine whether CK2 downregulation-induced SIRT1 destabilization was accompanied by ubiquitination, cells were treated with pcDNA3.1-HA-CK2 $\alpha$ or CK2 $\alpha$ siRNA. SIRT1 protein was immunoprecipitated from cells and then probed for ubiquitin using anti-ubiquitin antibodies, and higher levels of ubiquitinated SIRT1 were found in cells downregulating CK2 $\alpha$ compared with control cells. In contrast, ubiquitinated SIRT1 was reduced in cells overexpressing CK $2 \alpha$ compared with untreated controls (Fig. 3D). The level of ubiquitinated SIRT1 was increased by treatment with the proteasome inhibitor MG132 (data not shown). These observations indicate that the effect of CK $2 \alpha$ on reduction of SIRT1 protein was mainly a result of an increase in ubiquitination and proteosomal degradation of SIRT1.

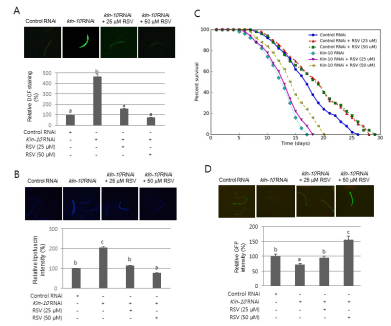

Fig. 4. Effect of the SIRT1 activator resveratrol on the lifespan shortening and age-related biomarkers mediated by kin-10 knockdown in C. elegans. Age-synchronized wild-type worms (A-C) and reporter strain sod-3::gfp (D) at the L4 stage were fed on the control RNAi plates or kin-10 RNAi plates containing 25 or $50 \mu \mathrm{M}$ resveratrol (RSV) for 3 days. All images were acquired at $10 \times$ magnification and fluorescence intensity was quantified using Image software by determining the average pixel intensity (A) Effect of resveratrol on ROS generation. Worms were incubated with DCFDA as described in the "Materials and methods" section. Representative fluorescence images (upper panel). The fluorescence intensity of nematodes ( $n=50$ per condition) (bottom panel). (B) Effect of resveratrol on lipofuscin accumulation. Representative fluorescence images (upper panel). The fluorescence intensity of lipofuscin ( $\mathrm{n}=50$ per condition) (bottom panel). (C) The effect of resveratrol on lifespan. Viability was scored as movement away from pick touch at the indicated days. Representative data from three independent RNAi experiments are shown ( $\mathrm{n}=50$ per condition). (D) Effect of resveratrol on DAF-16 activity. Representative fluorescence images (upper panel). Fluorescence intensity of GFP ( $n=50$ per condition) (bottom panel). 


\section{The SIRT1 activator resveratrol abrogates the lifespan shortening, lipofuscin accumulation, ROS generation, and reduced DAF-16 activity mediated by kin-10 knockdown in C. elegans}

Because C. elegans is a model for exploring the mechanisms underlying organism aging, our observation that SIRT1 is involved in ROS generation during CK2 downregulationmediated cellular senescence led us to examine the role of SIRT1 in nematode aging induced by CK2 downregulation. To analyze the role of SIRT1 in C. elegans aging after kin-10 knockdown, nematodes were incubated with the SIRT1 activator resveratrol. It has been previously reported that resveratrol stimulates the activity of purified SIR-2.1 (the ortholog of SIRT1) of C. elegans (19) and that the effect of resveratrol on nematode lifespan is completely dependent on SIR-2.1 (20). Consistent with our previous report (9), knockdown of kin-10 resulted in shorter lifespan and an increase in lipofuscin accumulation and ROS production compared to control RNAi (Fig. 4A-C). Treatment with resveratrol $(25$ or $50 \mu \mathrm{M})$ significantly abrogated ROS generation (Fig. 4A), lipofuscin accumulation (Fig. 4B), and lifespan shortening (Fig. 4C, Supplementary Fig. 5) mediated by kin-10 knockdown. DAF-16 (the ortholog of FoxO) is a central transcription factor, which detoxifies intracellular ROS by expressing antioxidant proteins in C. elegans $(21,22)$. Consistent with our previous report (9), kin-10 knockdown decreased the transcription of the sod-3::gfp reporter gene (Fig. 4D). Treatment with resveratrol significantly rescued the reduced transcriptional activity of DAF-16, which was mediated kin-10 knockdown (Fig. 4D). Collectively, these results suggest that SIRT1 is involved in nematode aging mediated by CK2 downregulation.

\section{DISCUSSION}

We have previously reported that CK2 is downregulated in senescent human lung fibroblast IMR-90 cells, aged rat tissues, and aging nematodes $(5-9,23)$. CK2 downregulation induces premature senescence in IMR-90, HCT116, and MCF-7 cells (23, 24), and kin-10 knockdown induces expression of age-related biomarkers and causes lifespan shortening in nematodes (9). CK2 downregulation stimulates AKT-mediated FoxO3a phosphorylation and nuclear export of FoxO3a, resulting in decreased transcription of antioxidant genes in human cells (8). CK2 downregulation increases ROS accumulation through downregulating DAF-16 activity in nematodes (9). The present study indicates that SIRT1 additionally plays an important role in CK2 downregulation- mediated ROS generation in both human cells and nematodes. In this study, SIRT1 overexpression suppressed both ROS production and reduction of FoxO3a transcriptional activity mediated by CK2 downregulation in HCT116 and MCF-7 cells (Fig. 1 and Supplementary Fig. 2A, B). In addition, SIRT1 overexpression attenuated both the nuclear export of FoxO3a and the acylation of FoxO3a induced by CK2 $\alpha$ downregulation in cells (Fig. 2). Consistent with these results, overexpression of a dominant-negative mutant SIRT1 (H363Y)
$(15,16)$, or treatment with nicotinamide suppressed the decreased ROS levels, reduction of FoxO3a activity, nuclear import of FoxO3a, and FoxO3a deacetylation induced by CK2 $\alpha$ upregulation in cells (Fig. 1, Fig. 2, Supplementary Fig. 1C, Supplementary Fig. 2C, and Supplementary Fig. 3). Furthermore, the SIRT1 activator resveratrol significantly abrogated the lifespan shortening, lipofuscin accumulation, ROS generation, and reduced DAF-16 activity mediated by kin-10 knockdown in nematodes (Fig. 4). Taken together, the present study suggests that CK2 downregulation may induce both cellular senescence and organism aging, at least in part, through downregulating the SIRT-FoxO3a axis. We have previously shown that CK2 downregulation induces acetylation and stabilization of $\mathrm{p} 53$ by negatively regulating SIRT1 deacetylase activity during senescence (18). Therefore, our findings to date demonstrate that SIRT1 deacetylase activity is involved in CK2 downregulation-induced senescence through the downstream effectors FoxO3a and p53, which regulate transcription of antioxidant genes and the CDK inhibitor p21 $1^{\text {Cip1/NAF1, }}$ respectively. Furthermore, the present study along with our previous findings suggest that in CK2 downregulation-induced senescence FoxO3a is regulated by the upstream modulators AKT and SIRT1, which modify FoxO3a by phosphorylation and deacetylation, respectively.

The previous studies have shown that CK2 promotes SIRT1 deacetylase activity through its phosphorylation $(17,18)$ and that phosphorylation of SIRT1 increases its substrate-binding affinity (17). The present study importantly indicates that CK2 downregulation stimulates degradation of SIRT1 protein through promoting SIRT1 ubiquitination in cells and, conversely, CK2 upregulation inhibits SIRT1 ubiquitination and increases the levels of SIRT1 protein. Ubiquitinated SIRT1 protein was increased by treatment with the proteasome inhibitor MG132, indicating that the proteasome pathway was responsible for degradation of SIRT1 protein induced by CK2 downregulation (Fig. 3). Collectively, the results found in this study and our previous work show that CK2 can modulate the activity and protein levels of SIRT1 through phosphorylation and ubiquitination. It has been reported that JNK1 and MEK1 signaling modulates SIRT1 stability through SIRT1 ubiquitination $(25,26)$. Ubiquitination is required for SIRT1 function during the DNA damage response (27). However, little is known about the molecular mechanism of SIRT1 ubiquitination. How does CK2 activity modulate the ubiquitination of SIRT1? Because it has been determined that $\mathrm{CK} 2$ phosphorylates E3 ubiquitin ligases such as MDM2 and $\mathrm{SCF}^{\text {(cyclin F) }}$ complex $(28,29)$, we speculate that CK2 regulates SIRT1 ubiquitination via CK2-mediated phosphorylation of SIRT1 and/or E3 ubiquitin ligases. Although further studies are required to identify more regulators for SIRT1 ubiquitination, the discovery of CK2 regulation of SIRT1 ubiquitination provides a new avenue of research in the fields of SIRT1 regulation and senescence. 


\section{MATERIALS AND METHODS}

\section{Cell culture, RNA interference, and DNA transfection}

HCT116 human colon cancer and MCF-7 human breast cancer cells were cultured in Dulbecco's modified Eagle medium containing $10 \%(\mathrm{v} / \mathrm{v})$ fetal bovine serum under a humidified atmosphere of $5 \%(\mathrm{v} / \mathrm{v}) \mathrm{CO}_{2}$ at $37^{\circ} \mathrm{C}$. siRNAs, pcDNA-HA-CK2 $\alpha$, pECE-Flag-SIRT1, and pECE-Flag-SIRT1 (H363Y) were transfected into cells using Lipofectamine (Invitrogen, Carlsbad, CA, USA) as described by the manufacturer's instruction. siRNA for CK2 $\alpha$ was 5'-UCAAGAUGACUACCAGCUGdTdT-3'. siRNA for the negative control was 5'-GCUCAGAUCAAUACGGAG AdTdT-3'. At $48 \mathrm{~h}$ after transfection, the cells were harvested.

\section{Measurement of intracellular ROS}

Intracellular ROS levels were measured as described previously (6).

\section{Western blotting}

Antibodies to CK2 $\alpha$, SIRT1, ubiquitin, and $\beta$-actin were obtained from Santa Cruz Biotechnology (Santa Cruz, CA, USA). Antibodies against FoxO3a and acetylated Lys were obtained from Cell Signaling Technology (Beverly, MA, USA). Western blotting was performed as described previously (6).

\section{Immunocytochemical staining}

Cells were seeded on four-well micro-chamber slides (Thermo Fisher Scientific, NY, USA) and fixed with $4 \%$ paraformaldehyde in phosphate-buffered saline (PBS) for $10 \mathrm{~min}$ at $25^{\circ} \mathrm{C}$ and permeabilized in $0.25 \%$ Triton X-100 before blocking with $2 \%$ bovine serum albumin in PBS. Primary antibodies (anti-FoxO3a; $1: 50$ ) were added at $25^{\circ} \mathrm{C}$ for $1 \mathrm{~h}$. The secondary antibodies were rhodamine-conjugated, goat anti-rabbit IgG (1:200, Invitrogen). Next, 4',6-diamidino-2- phenylindole (DAPI; Invitrogen) was used to counterstain nuclei and fluorescence signals were detected using a Carl Zeiss Axioplan 2 microscope (Carl Zeiss, Jena, Germany). Fluorescence images were analyzed using ImageJ software (National Institutes of Health, Bethesda, MD, USA; http://rsb.info.nih.gov/ij/).

\section{Reverse transcription-PCR}

Total RNA was extracted from HCT116 and MCF-7 cells. RNA was reverse transcribed using gene-specific primers and reverse transcriptase (Takara Bio Inc., Kyoto, Japan), and resulting cDNAs were PCR-amplified. The PCR primer sequences used for $\mathrm{Cu} / \mathrm{ZnSOD}, \mathrm{MnSOD}$, catalase, thioredoxin-2, peroxiredoxin-5, and $\beta$-actin have been reported previously (8). The PCR primer sequences used for SIRT1 were: forward (5'-GCAGATTAG TAGGCGGCTTG-3') and reverse (5'-TCTGGCATGTCCCACTA TCA-3'). PCR products were resolved on a $2 \%$ agarose gel. Quantification of the reverse transcribed-PCR bands was performed using densitometry. Levels of $\beta$-actin RNA were used to normalize the amount of RNA in each sample.

\section{Immunoprecipitation}

Cell lysates were pre-cleared with normal mouse or rabbit IgG and protein A sepharose beads (Amersham Pharmacia Biotech, Korea) for $1 \mathrm{~h}$ at $4^{\circ} \mathrm{C}$. The supernatant was then incubated with anti-acetylated Lys antibodies (Cell Signaling Technology, Danvers, MA, USA) and protein A sepharose beads with mixing for $12 \mathrm{~h}$ at $4^{\circ} \mathrm{C}$. Then, the beads were collected by centrifugation and washed three times with PBS.

\section{Culture of nematodes and RNAi experiment}

C. elegans N2 (wild-type) strain and reporter strains expressing sod-3::gfp were acquired from the Caenorhabditis Genetics Center. Nematodes were grown at $21^{\circ} \mathrm{C}$ on nematode growth medium (NGM) agar plates with Escherichia coli strain OP50 as a food source. For some experiments, nematodes were treated with resveratrol (Sigma-Aldrich, St. Louis, MO, USA). RNAi experiments were performed with $E$. coli $\mathrm{HT} 115$ cells expressing double-stranded kin-10 RNA, as described previously (9).

\section{Lifespan assays}

Lifespan assays were performed as described previously (30). Synchronized L4 larvae were placed on HT115-seeded NGM plates containing FUdR. Surviving nematodes were counted daily and were moved to fresh HT115-seeded NGM plates. Death was scored as the absence of a response to slight touch using a thin platinum wire. Three independent experiments were performed.

\section{Measurement of ROS and lipofuscin in nematodes}

ROS levels in synchronized (day 1 of adulthood) nematodes were measured as described previously (9). Intestinal lipofuscin accumulation in nematodes was determined by autofluorescence (9) using a fluorescence microscope (ZEISS AxioCam MRc, Jena, Germany) with excitation and emission wavelengths of $350 \mathrm{~nm}$ and $470 \mathrm{~nm}$, respectively. The relative fluorescence intensity was quantified using Imagej software to determine lipofuscin levels.

\section{Assays for reporter gene expression in nematodes}

Synchronized (L4 larva) nematodes expressing sod-3::gfp were transferred to HT115-seeded NGM plates. After 1 day, the fluorescence of GFP was measured using a fluorescence microscope at excitation and emission wavelengths of $490 \mathrm{~nm}$. The relative fluorescence intensity was quantified using ImageJ. Three independent experiments were performed.

\section{Statistical analysis}

Data were analyzed by one-way analysis of variance with the SPSS package program (IBM, Armonk, NY). The results were considered significant if the $\mathrm{P}$ value was $<0.05$. Duncan's multiple-range test was performed if the differences between the groups were identified as $\alpha=0.05$. For all bar graphs, bars that do not share a common letter (a, b, c) are significantly different among the groups at $\mathrm{P}<0.05$. 


\section{ACKNOWLEDGEMENTS}

This research was supported by Basic Science Research Program through the National Research Foundation of Korea (NRF) funded by the Ministry of Science, ICT, and Future Planning [grant number NRF-2015R1A2A2A01004593].

\section{CONFLICTS OF INTEREST}

The authors have no conflicting interests.

\section{REFERENCES}

1. Campisi J (2013) Aging, cellular senescence, and cancer. Annu Rev Physiol 75, 685-705

2. Rodier F and Campisi J (2011) Four faces of cellular senescence. J Cell Biol 192, 547-556

3. Kuilman T, Michaloglou C, Mooi WJ and Peeper DS (2010) The essence of senescence. Genes Dev 24, 2463-2479

4. Liou GY and Storz P (2010) Reactive oxygen species in cancer. Free Radic Res 44, 479-496

5. Kang JY, Kim JJ, Jang SY and Bae YS (2009) The p53-p21 ćip1/WAF1 pathway is necessary for cellular senescence induced by the inhibition of protein kinase CKII in human colon cancer cells. Mol Cells 28, 489-494

6. Jeon SM, Lee SJ, Kwon TK et al (2010) NADPH oxidase is involved in protein kinase CKII down-regulation-mediated senescence through elevation of the level of reactive oxygen species in human colon cancer cells. FEBS Lett 584, 3137-3142

7. Park JH, Kim JJ and Bae YS (2013) Involvement of PI3K-AKT-mTOR pathway in protein kinase CKII inhibition-mediated senescence in human colon cancer cells. Biochem Biophys Res Commun 433, 420-425

8. Park SY and Bae YS (2016) Inactivation of the FoxO3a transcription factor is associated with the production of reactive oxygen species during protein kinase CK2 downregulation-mediated senescence in human colon cancer and breast cancer cells. Biochem Biophys Res Commun 478, 18-24

9. Park JH, Lee JH, Park JW et al (2017) Downregulation of protein kinase CK2 activity induces age-related biomarkers in C. elegans. Oncotarget 8, 36950-36963

10. Storz $P$ (2011) Forkhead homeobox type $O$ transcription factors in the responses to oxidative stress. Antioxid Redox Signal 14, 593-605

11. Brunet A, Bonni A, Zigmond MJ et al (1999) Akt promotes cell survival by phosphorylating and inhibiting a Forkhead transcription factor. Cell 96, 857-868

12. Senf SM, Sandesara PB, Reed SA and Judge AR (2011) p300 Acetyltransferase activity differentially regulates the localization and activity of the FOXO homologues in skeletal muscle. Am J Physiol Cell Physiol 300, 1490-1501

13. Motta MC, Divecha N, Lemieux M et al (2004) Mammalian SIRT1 represses forkhead transcription factors. Cell 116, 551-563

14. Ferguson D, Shao N, Heller E et al (2015) SIRT1-FOXO3a regulate cocaine actions in the nucleus accumbens. J Neurosci 35, 3100-3111

15. Vaziri H, Dessain SK, Ng Eaton E et al (2001) hSIR2 (SIRT1) functions as an NAD-dependent p53 deacetylase. Cell 107, 149-159

16. van der Veer E, Ho C, O'Neil C et al (2007) Extension of human cell lifespan by nicotinamide phosphoribosyltransferase. J Biol Chem 282, 10841-1085

17. Kang H, Jung JW, Kim MK and Chung JH (2009) CK2 is the regulator of SIRT1 substrate-binding affinity, deacetylase activity and cellular response to DNA-damage. PLoS One 4, e6611

18. Jang SY, Kim SY and Bae YS (2011) p53 deacetylation by SIRT1 decreases during protein kinase CK2 downregulationmediated cellular senescence. FEBS Lett 585, 3360-3366

19. Wood JG, Rogina B, Lavu S et al (2004) Sirtuin activators mimic caloric restriction and delay ageing in metazoans. Nature 430, 686-689

20. Viswanathan M, Kim SK, Berdichevsky A and Guarente L (2005) A role for SIR-2.1 regulation of ER stress response genes in determining C. elegans life span. Dev Cell 9, 605-615

21. Murphy CT, McCarroll SA, Bargmann Cl et al (2003) Genes that act downstream of DAF-16 to influence the lifespan of Caenorhabditis elegans. Nature 424, 277-283

22. Ogg S, Paradis S, Gottlieb S et al (1997) The Fork head transcription factor DAF-16 transduces insulin-like metabolic and longevity signals in C. elegans. Nature 389, 994-999

23. Ryu SW, Woo JH, Kim YH et al (2006) Downregulation of protein kinase CKII is associated with cellular senescence. FEBS Lett 580, 988-994

24. Lee YH, Yuk HJ, Park KH and Bae YS (2013) Coumestrol induces senescence through protein kinase CKII inhibition-mediated reactive oxygen species production in human breast cancer and colon cancer cells. Food Chem 141, 381-388

25. Hwang JS, Ham SA, Yoo T et al (2016) Upregulation of MKP-7 in response to rosiglitazone treatment ameliorates lipopolysaccharide-induced destabilization of SIRT1 by inactivating JNK. Pharmacol Res 114, 47-55

26. Cheng J, Liu C, Liu L et al (2016) MEK1 signaling promotes self-renewal and tumorigenicity of liver cancer stem cells via maintaining SIRT1 protein stabilization. Oncotarget 7, 20597-20611.

27. Peng L, Yuan Z, Li Y et al (2015) Ubiquitinated sirtuin 1 (SIRT1) function is modulated during DNA damageinduced cell death and survival. J Biol Chem 290, 8904-8912

28. Allende-Vega N, Dias S, Milne D and Meek D (2005) Phosphorylation of the acidic domain of $\mathrm{Mdm} 2$ by protein kinase CK2. Mol Cell Biochem 274, 85-90

29. Lee A, Rayner SL, De Luca A et al (2017) Casein kinase II phosphorylation of cyclin $\mathrm{F}$ at serine 621 regulates the Lys48-ubiquitylation E3 ligase activity of the SCF ${ }^{\text {(cyclin F }}$ complex. Open Biol 7, 170058

30. Lee SJ, Hwang AB and Kenyon C (2010) Inhibition of respiration extends $C$. elegans life span via reactive oxygen species that increase HIF-1 activity. Curr Biol 20, 2131-2136 